\title{
Patient-Centered Medical Home Implementation in the Veterans Health Administration and Primary Care Use: Differences by Patient Comorbidity Burden
}

\author{
Edwin S. Wong, Ph.D. ${ }^{1,2}$, Ann-Marie Rosland, M.D. M.S.3,4, Stephan D. Fihn, M.D. M.P.H. ${ }^{5}$, \\ and Karin M. Nelson, M.D. M.S.H.S. ${ }^{1,2,6}$ \\ 'VA Puget Sound Health Care System, Center for Veteran-Centered and Value-Driven Care, Seattle, WA, USA; ${ }^{2}$ Department of Health Services, \\ University of Washington, Seattle, WA, USA; ${ }^{3}$ Center for Clinical Management Research, VHA Ann Arbor Healthcare System, Ann Arbor, MI, USA; \\ ${ }^{4}$ Department of Internal Medicine, University of Michigan, Ann Arbor, MI, USA; ${ }^{5}$ Office of Analytics and Business Intelligence, Veterans Health \\ Administration, Seattle, WA, USA; ${ }^{6}$ Department of Medicine, University of Washington, Seattle, WA, USA.
}

BACKGROUND: The patient-centered medical home (PCMH) model has several components to improve care for patients with high comorbidity, including greater access to face-to-face primary care.

OBJECTIVE: We examined whether high-comorbidity patients had larger increases in primary care provider (PCP) visits attributable to $\mathrm{PCMH}$ implementation in a large integrated health system relative to other patients enrolled in primary care.

DESIGN, SUBJECTS AND MAIN MEASURES: This longitudinal study examined a $1 \%$ random sample of 9.3 million patients enrolled in the Veterans Health Administration (VHA) at any time between 2003 and 2013. Faceto-face visits with PCPs per quarter were identified through VHA administrative data. Comorbidity was measured using the Gagne index and patients with a weighted score of $\geq 2$ were defined as high comorbidity. We applied interrupted time-series models to estimate marginal changes in PCP visits attributable to PCMH implementation. Differences in marginal changes were calculated across comorbidity groups (high vs. low). Analyses were stratified by age group to account for Medicare eligibility. KEY RESULTS: Among age 65+ patients, PCMH was associated with greater PCP visits starting four and ten quarters following implementation for high- and lowcomorbidity patients, respectively. Changes were larger for high-comorbidity patients (eight to 11 greater visits per 1000 patients per quarter). Among patients age < 65, $\mathrm{PCMH}$ was associated with greater visits for highcomorbidity patients starting eight quarters following implementation, but fewer visits for low-comorbidity patients in all quarters. The difference in visit changes across groups ranged from 18 to 67 visits per 1000 patients per quarter. CONCLUSIONS: Increases in PCP visits attributable to $\mathrm{PCMH}$ were greater among patients with higher comorbidity. Health systems implementing PCMH should account for population-level comorbidity burden when planning for PCMH-related changes in PCP utilization.

Electronic supplementary material The online version of this article (doi:10.1007/s11606-016-3833-9) contains supplementary material, which is available to authorized users.

Received January 29, 2016

Revised May 27, 2016

Accepted July 22, 2016

Published online August 8, 2016
KEY WORDS: patient-centered medical home; multiple chronic conditions; primary care; utilization.

J Gen Intern Med 31(12): 1467-74

DOI: $10.1007 / \mathrm{s} 11606-016-3833-9$

c Society of General Internal Medicine 2016

\section{INTRODUCTION}

In April 2010, the Veterans Health Administration (VHA) began to establish patient-centered medical homes (PCMHs) at all primary care clinics nationwide as part of the Patient Aligned Care Team (PACT) initiative. PACT represents the largest PCMH implementation to date in the United States and is based on provision of team-based care that is accessible, coordinated, comprehensive and patient-driven. ${ }^{1-3}$ PACT builds upon previous system level transformations implemented by VHA, such as adoption of the electronic medical record, a marked expansion of primary care services and the creation of Veterans Integrated Service Networks to support care coordination and that share a global budget. ${ }^{4}$

Several PACT components could affect use of primary care services. Notably, efforts to improve primary care access included expanding clinic staffing, increasing the availability of same-day appointments, promoting nurse appointments and facilitating virtual encounters by phone, secure messaging and telehealth. In addition, the PACT focus on restructuring the delivery of primary care through teams may improve clinics' efficiency. ${ }^{5-7}$ PACT provisions to increase access and integrate team-based care could increase capacity and the frequency of primary care provider (PCP) visits for some patients, particularly those with a relatively high comorbidity burden and a greater need for face-to-face primary care. ${ }^{8-10}$ Finally, PACT efforts to increase care coordination, including the shifting of some services from specialists to primary care, are likely to disproportionately affect high-comorbidity patients, particularly those with multiple providers or who are dually enrolled in Medicare. ${ }^{11,12}$ In contrast, healthier primary care patients might have decreased use of face-to-face primary care if other options introduced by PACT, such as telephone or electronic visits, served as an appropriate substitute. 
Prior studies have yielded variable findings regarding whether PCMH implementation was associated with changes in frequency of primary care visits, but none stratified patients according to comorbidity. ${ }^{13-15}$ We sought to examine whether changes in the frequency of face-to-face primary care provider (PCP) visits following implementation of the PCMH model in VHA differed according to patients' comorbidity burden. This study contributes to the growing literature examining sources of heterogeneity in PCMH effects on utilization patterns. Additionally, this study addresses the question of whether increased primary care resources provided to patients by PACT are being focused on those with the highest health care needs.

\section{METHODS}

\section{Data and Study Sample}

Data for this study were derived from the VHA Corporate Data Warehouse (CDW), which contains comprehensive administrative data tracking patient utilization, demographics and clinical measures including ICD-9 diagnostic codes. Using the Primary Care Management Module (PCMM) within CDW, we identified 9.3 million patients who received care from VHA between October 2003 and September 2013 (fiscal year [FY] 2004 to FY2013). PCMM tracks patients with any VHA health care use during the preceding 24 months. We partitioned the follow-up period into 40 quarters. For each quarter, we identified a $1 \%$ random sample of all VHA primary care patients present in PCMM in that quarter. The final study sample consisted of 8.4 million patient-quarter observations. All analyses were stratified by age group (under 65 and age $65+$ ) because nearly all patients age $65+$ who use VHA primary care are simultaneously eligible for non-VHA primary care through Medicare.

\section{Patient Comorbidity Measure}

We measured patient comorbidity burden using the Gagne (2011) comorbidity score. ${ }^{16}$ The Gagne measure is a weighted composite of conditions from the Charlson and Elixhauser comorbidity measures with improved performance in predicting 30-day mortality compared to the component measures. We categorized patients with a Gagne score $\geq 2$ as having high comorbidity burden, which corresponds to roughly the $85^{\text {th }}$ percentile of the Gagne score distribution among all patients enrolled in VA primary care at the time of PACT implementation (FY2010Q3).

\section{Outcome Measure}

Outpatient visit measures were based on VHA encounter stop codes that indicate the main clinic group responsible for providing a set of specific health services (e.g., primary care, mental health, pulmonology, etc.). We ascertained the number of face-to-face PCP visits to clinics identified as providing primary care during each 3-month interval. We included visits to all provider types including medical doctors, nurse practitioners and physician assistants.

\section{Statistical Analysis}

We applied interrupted time series (ITS) models to examine associations between PACT implementation and PCP visit counts within VHA. ${ }^{17}$ ITS models were used because PACT was simultaneously implemented across all VHA clinics in April 2010; therefore, no control group was present to compare PCP visit changes following PACT. The goal of ITS models is to derive long-run time trends in quarterly PCP visits and identify whether deviations from long-run temporal trends exist following PACT implementation. The ITS models we estimated were an intent-to-treat analysis, providing inferences about the transition to a PACT model of primary care delivery.

We estimated ITS models using negative binomial regression to account for right skewness in the distribution of PCP visits. To model potential trend deviations, we included three variables: 1) an indicator variable denoting observations that followed implementation of PACT; 2) a non-linear time trend term; and 3) an interaction between the post-PACT indicator and the time-trend term. We then modeled potential differences in trend deviations following the start of PACT implementation in relation to patient comorbidity by including: 1) the three variables described above; 2) an indicator for high comorbidity; 3) two-way interactions between the high-comorbidity indicator, the post-PACT indicator and the non-linear time trend, respectively; and 4) the three-way interaction between these variables. The best fitting functional form for the non-linear time trend term, identified using fractional polynomials, was a quadratic function for the age $<65$ population and a reciprocal function (i.e., $1 / t$ ) for the age $65+$ population. ${ }^{18}$

Regression models adjusted for patient age, gender, marital status, race/ethnicity (White, Black, Hispanic or other), education, eligibility for copayment-exempt VHA care, distance from patients' residence to the nearest VHA clinic, indicator variables for the presence of comorbidities and an indicator for hospitalization in the prior 12 months. Additionally, we adjusted for the number of PCP visits in the prior 12 months as a proxy for unobserved patient variables predictive of PCP use. Because the observation period coincided with the Great Recession, we also adjusted for the unemployment rate in patients' residence county to account for PCP visit changes influenced by economic conditions. ${ }^{19,20}$ The unit of analysis for regression models was patient-quarter.

Using estimated parameter values, we calculated the expected number of PCP visits for each quarter after April 2010 conditional on high-comorbidity burden, PACT being implemented (post-PACT $=1$ ) and holding all covariates at their respective sample means. We repeated this calculation conditional on PACT not being implemented (post-PACT $=0$ ). We then calculated the "PACT association" as the difference in expected PCP visits with and without PACT for each quarter. The PACT association was computed in a similar manner for 
low-comorbidity patients by holding high comorbidity $=0$. Finally, we calculated the difference in PACT associations between high- and low-comorbidity patients for each quarter. Standard errors from all regression models were heteroskedastic robust. The delta method was applied to generate standard error estimates for model predictions described above. ${ }^{21}$ All statistical analyses were performed using STATA version 12.0 (STATA Corp., College Station, TX). A nominal $p$ value of 0.05 was used to assess all statistical hypotheses.

\section{Sensitivity Analysis}

We conducted two sensitivity analyses. First, the Gagne measure was validated among patients age $65+$, so we performed sensitivity analyses using the Elixhauser measure, which has been validated among patients of all ages. ${ }^{22,23}$ We estimated separate models categorizing patients with an Elixhauser score of $\geq 4$ and $\geq 5$ (i.e., presence of four or five Elixhauser comorbidities), respectively, as high comorbidity. These cutoffs corresponded to approximately the $85^{\text {th }}$ and $92^{\text {nd }}$ percentile of scores among patients in FY2010Q3. Results from analyses based on the Elixhauser measure were similar to the primary findings (Online Appendix, Figs. 1-4). Second, we estimated ITS models among the subgroup of patients exempt from VHA copayments to assess whether potential changes in PCP use among high comorbidity patients were driven by eliminated cost sharing. The magnitude of ITS model estimates from these analyses was similar to the primary results (Online Appendix, Figures 5-6).

\section{RESULTS}

\section{Descriptive Statistics}

Patients who were 65 years and older at the time of PACT implementation had an average age of 76.6 years and most were male (98.1\%), exempt from VHA copayments (73.0 \%), married $(67.0 \%)$ and White $(85.1 \%)$ (Table 1$)$. Nineteen percent of these elderly patients were classified as high comorbidity (Gagne score $\geq 2$ ).

Among age $<65$ patients, mean age was 52.0 years and most were male $(89.7 \%)$, exempt from copayments for VHA care $(87.4 \%)$ and White $(64.0 \%)$. Approximately $11 \%$ of patients younger than 65 years met the definition of high comorbidity. High-comorbidity patients in both age groups were more likely to be exempt from VHA copayments, Black and unmarried. High-comorbidity patients were also more likely to have been hospitalized and to have made more PCP visits in the 12-months prior to PACT implementation than lower-comorbidity patients.

\section{Unadjusted Results}

PCP visit trends were dominated by seasonality among all patient subgroups, with spikes occurring during October-December each year (Fig. 1). Among high-comorbidity patients
65 years and older, average quarterly PCP use increased $5 \%$ from 1198 visits per 1000 patients before PACT was initiated to 1255 visits per 1000 afterward. Among low-comorbidity patients in this age group, average quarterly PCP use increased only $2 \%$ from 716 to 729 visits per 1000 . For highcomorbidity patients under 65 years, average quarterly PCP use increased $10 \%$ from 1310 to 1440 visits per 1000 . Among low-comorbidity patients under 65, average quarterly PCP use increased $3 \%$ from 757 to 783 visits per 1000 patients. Lower VHA PCP use among older patients who were compared likely reflected better access to non-VHA primary care through Medicare.

\section{PACT Implementation and PCP Visits Among Age 65+ Patients}

Based on ITS models, PACT was associated with greater PCP use in VHA (Fig. 2). Among high-comorbidity patients, the increase in use attributable to PACT was statistically significant beginning four quarters after PACT implementation (six additional visits per 1000 patients, $p=0.047$ ) and increased to 18 additional visits per 1000 patients $(p<0.001)$ at the end of the observation period (13 quarters after PACT, FY2013Q4). Among low-comorbidity patients, PACT was associated with an initial decrease in PCP use, but was subsequently associated with greater PCP use beginning ten quarters after PACT implementation (five additional visits per 1000 patients, $p=$ 0.029). The change in PCP use associated with PACT had increased to eight additional visits per 1000 patients $(p=$ 0.004 ) by the end of the observation period.

The difference in PCP visit changes associated with PACT between high- and low-comorbidity patients was positive in all quarters, indicating relatively greater VHA PCP use associated with PACT among high-comorbidity patients (Fig. 3). The magnitude of this difference ranged from eight to 11 visits per 1000 patients and was statistically significant between two and 12 quarters following PACT implementation.

\section{PACT Implementation and PCP Visits Among Age $<65$ Patients}

Among patients younger than 65, PACT implementation was associated with an initial decrease in PCP use among highcomorbidity patients (19 fewer visits per 1000 patients, $p=$ 0.003). However, PACT implementation was associated with greater PCP use starting eight quarters after initial implementation (21 additional visits per 1000 patients, $p=0.023$ ) and through the end of the observation period (56 additional visits per 1000 patients, $p<0.001$ ).

Among low-comorbidity patients, we calculated an initial 17 visit per 1000 patients decrease $(p<0.001)$ in PCP use attributable to PACT (Fig. 2). The decline in use attenuated to 11 fewer visits per 1000 patients $(p=0.062)$ at the end of observation. The difference in PCP visit changes associated with PACT between high- and low-comorbidity patients was statistically significant starting five quarters after 
Table 1. Characteristics of VHA Patients at PACT Initial Implementation (April 2010) by Patient Comorbidity Burden

\begin{tabular}{|c|c|c|c|c|c|c|}
\hline \multirow[t]{2}{*}{ Variable } & \multicolumn{3}{|l|}{ Age 65+ } & \multicolumn{3}{|l|}{ Age $<65$} \\
\hline & $\begin{array}{l}\text { Overall } \\
N=120,337\end{array}$ & $\begin{array}{l}\text { Low-comorbidity } \\
N=97,152\end{array}$ & $\begin{array}{l}\text { High-comorbidity } \\
N=\mathbf{2 3}, \mathbf{1 8 5}\end{array}$ & $\begin{array}{l}\text { Overall } \\
N=128,989\end{array}$ & $\begin{array}{l}\text { Low-comorbidity } \\
N=114,984\end{array}$ & $\begin{array}{l}\text { High-comorbidity } \\
N=\mathbf{1 4 , 0 0 6}\end{array}$ \\
\hline Age (mean/sd) & $77(8)$ & $76(8)$ & $78(8)$ & $52(11)$ & $52(12)$ & $56(8)$ \\
\hline Male $(\%)$ & 98.1 & 98.0 & 98.4 & 89.7 & 89.5 & 90.9 \\
\hline $\begin{array}{l}\text { Exempt from VHA } \\
\text { Copayments (\%) }\end{array}$ & 73.0 & 70.9 & 81.6 & 87.4 & 86.5 & 94.5 \\
\hline Married (\%) & 67.0 & 68.2 & 62.2 & 48.4 & 49.2 & 41.9 \\
\hline \multicolumn{7}{|l|}{ Race $(\%)$} \\
\hline White & 85.1 & 85.7 & 82.7 & 64.0 & 63.6 & 66.7 \\
\hline Black & 8.1 & 7.5 & 10.6 & 20.2 & 20.1 & 21.4 \\
\hline Other & 6.8 & 6.9 & 6.7 & 15.8 & 16.3 & 11.9 \\
\hline Miles to Nearest VHA & $13(14)$ & $13(14)$ & $13(14)$ & $12(15)$ & $12(16)$ & $12(14)$ \\
\hline \multicolumn{7}{|c|}{ Comorbidities in Gagne Index (\%) } \\
\hline Cancer & 0.6 & 0.0 & 3.2 & 0.4 & 0.0 & 3.4 \\
\hline Congestive Heart Failure & 6.9 & 0.9 & 32.1 & 2.6 & 0.4 & 20.9 \\
\hline Dementia & 1.9 & 0.5 & 8.1 & 0.1 & 0.0 & 1.2 \\
\hline Renal Failure & 8.3 & 1.8 & 35.5 & 2.6 & 0.6 & 19.2 \\
\hline Weight Loss & 0.2 & 0.0 & 1.2 & 0.2 & 0.0 & 1.6 \\
\hline Hemiplegia & 0.5 & 0.3 & 1.5 & 0.7 & 0.5 & 2.7 \\
\hline Alcohol Abuse & 0.7 & 0.4 & 2.2 & 3.9 & 2.0 & 19.4 \\
\hline Any Tumor & 14.2 & 10.4 & 30.1 & 4.6 & 3.1 & 17.4 \\
\hline Arrhythmia & 13.2 & 7.7 & 36.0 & 4.0 & 2.1 & 19.4 \\
\hline $\begin{array}{l}\text { Chronic Pulmonary } \\
\text { Disease }\end{array}$ & 16.1 & 11.2 & 36.8 & 11.8 & 8.4 & 39.7 \\
\hline Coagulopathy & 1.6 & 0.7 & 5.3 & 0.9 & 0.3 & 5.4 \\
\hline Complicated Diabetes & 7.1 & 4.3 & 18.8 & 5.0 & 3.3 & 18.9 \\
\hline Anemia & 9.4 & 4.9 & 28.2 & 4.4 & 2.4 & 20.6 \\
\hline Fluid/Electrolyte Disorder & 3.5 & 1.3 & 12.5 & 2.7 & 1.2 & 15.0 \\
\hline Liver Disease & 0.9 & 0.5 & 2.6 & 3.5 & 2.0 & 15.5 \\
\hline $\begin{array}{l}\text { Peripheral Vascular } \\
\text { Disorder }\end{array}$ & 8.3 & 4.9 & 22.3 & 2.9 & 1.7 & 13.0 \\
\hline Psychosis & 4.6 & 2.9 & 11.6 & 15.2 & 11.7 & 43.8 \\
\hline $\begin{array}{l}\text { Pulmonary Circulation } \\
\text { Disorders }\end{array}$ & 1.0 & 0.3 & 3.9 & 0.6 & 0.2 & 3.9 \\
\hline HIV/AIDS & 0.1 & 0.1 & 0.1 & 0.7 & 0.7 & 0.6 \\
\hline Hypertension & 67.0 & 67.6 & 64.5 & 45.4 & 44.9 & 49.4 \\
\hline Cerebrovascular Disease & 7.9 & 6.4 & 14.3 & 3.1 & 2.5 & 8.4 \\
\hline Depression & 7.9 & 6.7 & 12.9 & 17.8 & 16.3 & 30.0 \\
\hline Myocardial Infarction & 2.2 & 1.5 & 4.9 & 1.1 & 0.8 & 3.8 \\
\hline $\begin{array}{l}\text { Neurodegenerative } \\
\text { Disorders }\end{array}$ & 5.7 & 4.3 & 11.6 & 4.1 & 3.4 & 9.3 \\
\hline Obesity & 12.1 & 11.6 & 14.5 & 19.3 & 18.6 & 24.8 \\
\hline Paralysis & 0.9 & 0.6 & 2.3 & 0.9 & 0.6 & 3.3 \\
\hline Rheumatoid Arthritis & 1.4 & 1.3 & 2.0 & 0.8 & 0.8 & 1.4 \\
\hline Drug Abuse & 0.5 & 0.3 & 1.1 & 5.1 & 3.9 & 14.7 \\
\hline Hypothyroidism & 8.6 & 7.8 & 12.0 & 4.5 & 4.1 & 7.5 \\
\hline Ulcer Disease & 1.2 & 1.0 & 1.8 & 0.7 & 0.6 & 1.7 \\
\hline Uncomplicated Diabetes & 28.5 & 25.9 & 39.6 & 19.1 & 17.3 & 34.5 \\
\hline Valvular Disease & 3.8 & 2.7 & 8.3 & 1.3 & 0.9 & 4.2 \\
\hline $\begin{array}{l}\text { Hospitalized Prior } \\
\text { Year }(\%)\end{array}$ & 6.6 & 3.4 & 19.8 & 7.9 & 5.1 & 30.3 \\
\hline $\begin{array}{l}\text { \# Primary Care Provider } \\
\text { Visits Prior Year } \\
(\mathrm{mean} / \mathrm{sd})\end{array}$ & $3.4(3.5)$ & $3.0(2.9)$ & $5.0(4.8)$ & $3.4(3.8)$ & $3.1(3.4)$ & $5.8(5.6)$ \\
\hline
\end{tabular}

$F Y=$ fiscal year, $Q=$ quarter, $V H A=$ Veterans Health Administration

Differences between low and high comorbidity patients significant at $5 \%$ for all variables, except HIV/AIDS among age $<65$ patients

High-comorbidity patients defined at those with Gagne risk score $>=2$

implementation ( 18 visits per 1000 patients, $p=0.019$ ) and increased through the end of the observation period (67 visits per 1000 patients, $p<0.001$ ) (Fig. 3).

\section{DISCUSSION}

Based upon extensive data from a large, national, integrated health system, we observed a significant increase in the use of
PCP visits among patients age $65+$, especially among relatively high-comorbidity patients, that could be attributed to implementation of a PCMH model of care. Among highcomorbidity patients, the increased use of face-to-face primary care within VHA grew over time, although the net change was modest overall. The strongest association was identified 13 quarters after PACT implementation (FY2013-Q4) and translated into $1.4 \%$ of the average. In the age $<65$ population, we observed an analogous positive and increasing association 

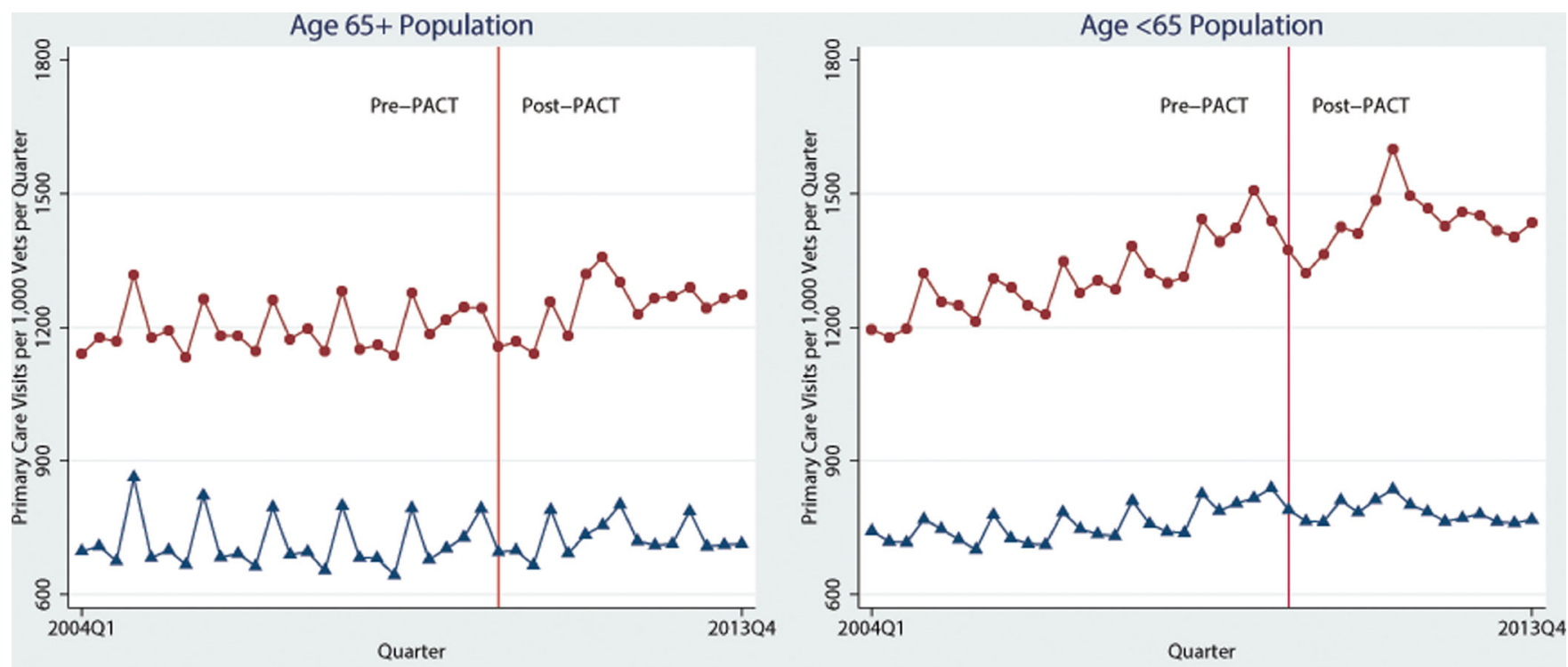

\section{$\longrightarrow$ Low Comorbidity High Comorbidity}

Figure 1. Unadjusted trends in VHA primary care provider visits by patient comorbidity. Data points reflect average VHA primary care provider visits per 1000 patients per quarter.

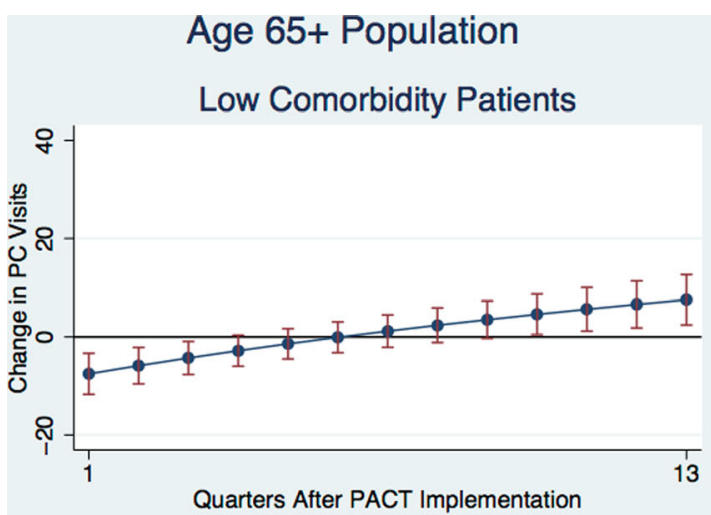

High Comorbidity Patients

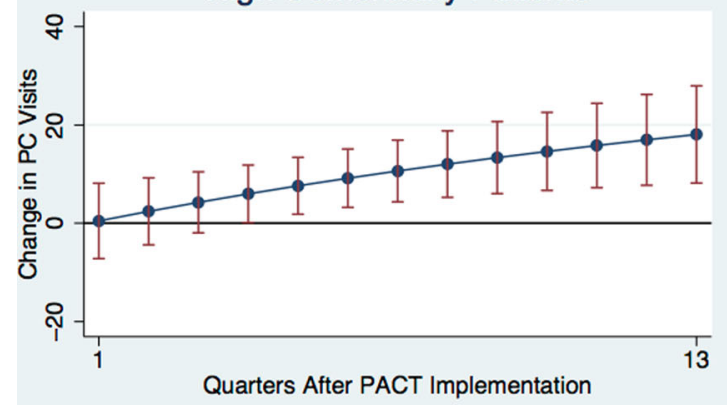

Age $<65$ Population

Low Comorbidity Patients

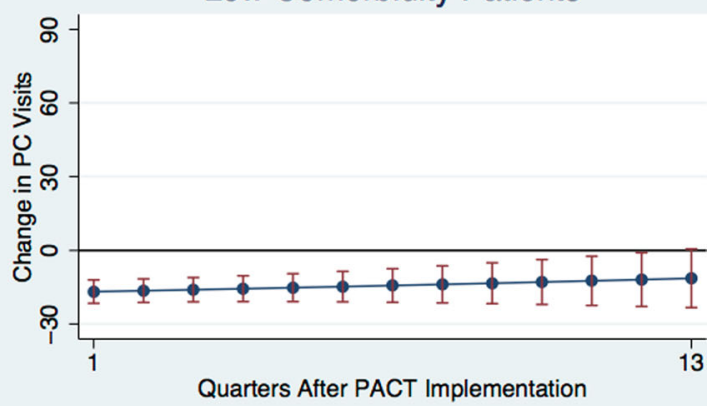

High Comorbidity Patients

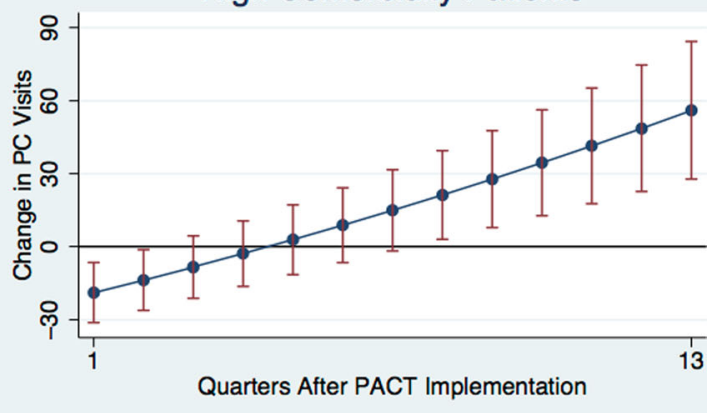

\section{$\longrightarrow$ Change in PC Visits $\longmapsto 95 \% \mathrm{Cl}$}

Figure 2. Association between PACT implementation and quarterly primary care provider visits by patient comorbidity. Data points reflect the change in VHA primary care provider visits associated with PACT for high-comorbidity patients minus the analogous change for lowcomorbidity patients. The high-comorbidity group consisted of patients with a Gagne score $\geq 2$. The low-comorbidity group consisted of all other patients. 


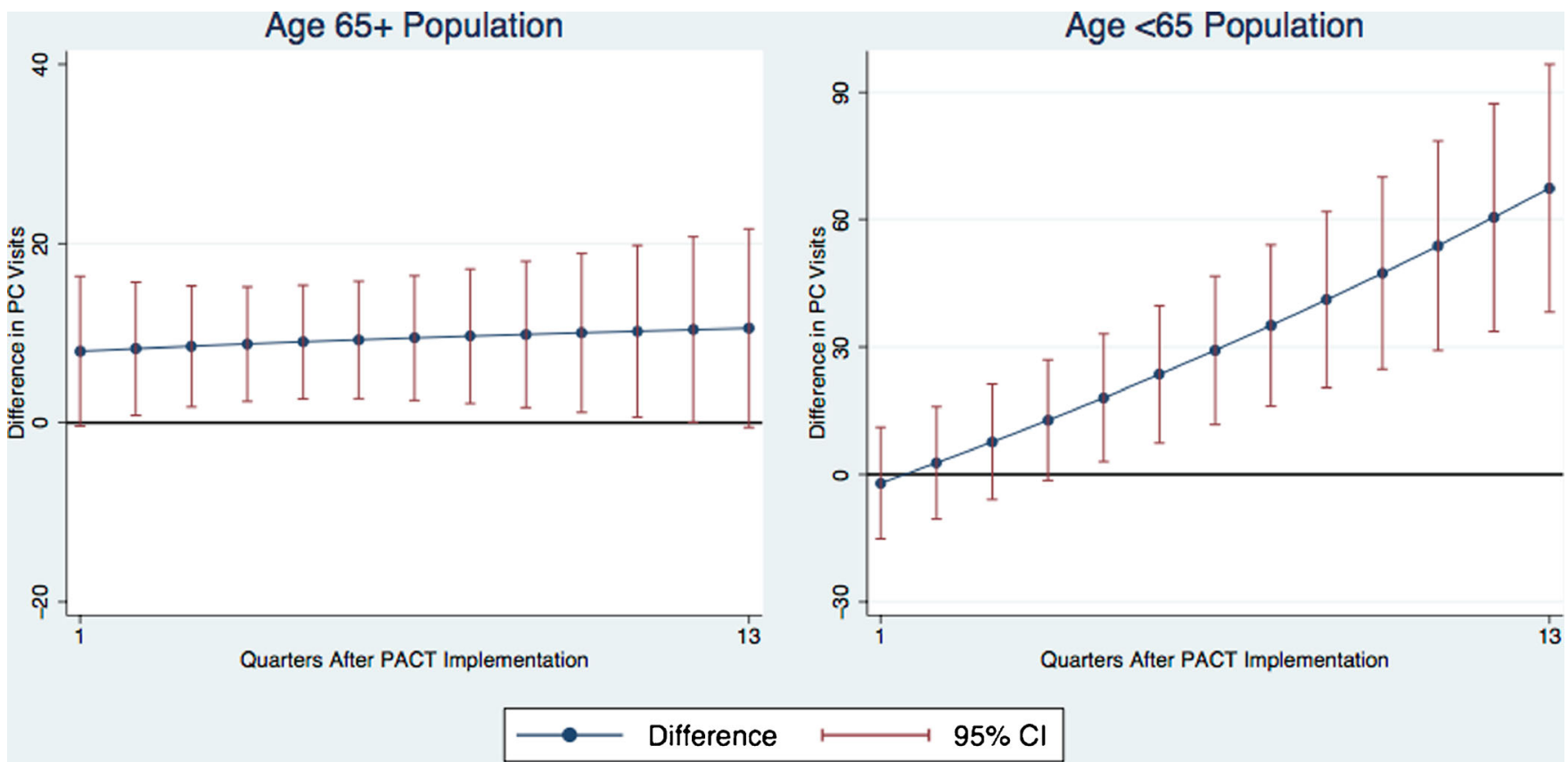

Figure 3. Difference in the association between PACT implementation and VHA primary care provider visits. Data points reflect the change in VHA primary care provider visits associated with PACT for high-comorbidity patients minus the analogous change for low-comorbidity patients. The high-comorbidity group consisted of patients with a Gagne score $\geq 2$. The low-comorbidity group consisted of all other patients.

between PACT implementation and PCP visits, which translated into $3.9 \%$ of the average at the peak, 13 quarters after PACT implementation. In contrast, the introduction of PACT was associated with a roughly $2 \%$ decline in face-to-face primary use among younger low-comorbidity patients, which remained constant during the 3-year post-PACT period.

PACT implementation had differing effects on face-to-face PCP visits varying by patients' level of comorbidity. Among the age $65+$ population, our results suggest that patients with a relatively high comorbidity burden and likely a greater need for face-to-face encounters were affected the most by increased access to primary care services, greater clinic efficiency resulting from team-based care and increased emphasis on care coordination by PCPs through PACT. Prior research by Rosland and colleagues demonstrated a $30 \%$ increase in VHA primary care staffing levels between April 2009 and April 2012. ${ }^{9}$ Additionally, a $9 \%$ increase in accommodation of same-day appointments requests and a $6 \%$ increase in the proportion of patients seen within 7 days of the desired appointment date were observed during the same period.

In contrast, the decrease in face-to-face primary care use associated with PACT for younger patients, particularly among relatively healthy patients, was potentially due to an appropriate uptake of multi-disciplinary care by nurses or other providers and substitutes to face-to-face care such as telephone care, electronic messaging and enhanced personal health record use. Use of phone encounters in particular increased nearly 11-fold overall between April 2009 and April 2012. ${ }^{9}$ Therefore, the reduction in face-to-face visits associated with PACT among younger and healthier patients may not reflect a net decrease in PCP workload, because PACT also likely increased non-face-to-face care among these patients. We were unable to examine changes in non-face-to-face care attributable to PACT in this study because complete data tracking these measures were not available over the 10-year study period. Future research should examine whether various primary care resources available through PCMH are being differentially allocated based on patient care needs in an appropriate manner.

To our knowledge, this is the first study to examine whether changes in primary care use following PCMH implementation differed by level of patient comorbidity. Three notable studies have examined the association between PCMH and face-toface primary care use with mixed results. Our results correspond to prior research that found PACT implementation was associated with a significant increase in primary care visits among veterans age $65+$ years, but no change among younger veterans after 2 years of follow-up. ${ }^{14}$ Reid and colleagues identified significant decreases in face-to-face primary care utilization within the Group Health Medical Home at 12, 18 and 24 months following implementation. ${ }^{15}$ In a more recent evaluation of the Southeastern Pennsylvania Chronic Care Initiative, participation in the medical home pilot by primary care practices was not significantly associated with changes in primary care use, relative to comparison practices over the 3year intervention period. ${ }^{13}$ However, the absence of changes in primary care use was potentially due to comparison practices simultaneously adopting PCMH elements during the pilot. There are at least two potential reasons why the association between PCMH and primary care utilization differed among these prior studies. First, although each study measured face-to-face primary care visits, the exact definition differed. 
Differences in findings across these studies may also be due to differences in clinical complexity of the underlying patient populations, which were not accounted for in these studies.

Disproportionate changes in utilization outcomes following implementation of PCMH among patients with highcomorbidity burden have been identified in prior studies, but these studies did not assess primary care utilization. ${ }^{24-27}$ Flottemesch and colleagues found PCMH clinical practice systems were associated with lower inpatient and emergency department use, as well as lower outpatient and total costs only among patients taking seven or more medications. ${ }^{25}$ Higgins and colleagues found non-significant associations between adoption of PCMH and total costs, and hospitalization overall masked significant reductions among patients in the highest risk decile. ${ }^{26}$

This study should be interpreted in the context of its limitations. First, implementation of PACT builds upon prior VHA investments in infrastructure and health information technology necessary to meet PCMH goals. ${ }^{8}$ Thus, interpretation of study findings should consider differences between PACT and implementation of other medical homes recognized by the National Committee for Quality Assurance. Second, our methodological approach applied ITS models, because PACT implementation occurred simultaneously in all VHA clinics and a natural control group of non-PACT clinics did not exist. Estimation of ITS models in this study measured changes in PCP visits attributable to system-wide PACT implementation, instead of the specific effect of PACT components. Third, estimation of changes in PCP visits attributable to PACT assumed that patient covariates, such as clinical conditions, were the same had PACT not been implemented. This assumption is likely to be less problematic over a shorter follow-up period. Finally, our ITS models were designed to attribute changes in PCP visits to PACT implementation, a substantial change to primary care delivery targeted to all VHA clinics nationwide. Other system-wide policy changes concurrent to PACT implementation may potentially confound estimates derived in this study. However, such policy changes are likely to be substantially smaller in scale relative to PACT.

\section{CONCLUSION}

In this study, implementation of the PCMH model of primary care delivery in VHA through the PACT initiative was associated with a modest increase in PCP visits among age 65+ patients, which materialized over time. Although all age 65+ patients appeared to increase use of face-to-face primary care, the change was disproportionately larger among relatively high-comorbidity patients. For age $<65$ VHA patients, PACT implementation was associated with an increase in PCP visits for high-comorbidity patients and a decrease in visits for lowcomorbidity patients. Health systems implementing PCMH may need to plan for a more marked increase in PCP utilization among their patients with greater comorbidity burden, which may be offset by decreases in other more costly types of utilization.

Acknowledgements: This study was funded by the Veterans Health Administration Patient Aligned Care Team Demonstration Laboratory Coordination Center (XVA-61-041). Dr. Wong is supported by a VA Health Services Research and Development Career Development Award (VA HSR\&D CDA 13-024). Dr. Rosland is supported by VA HSR\&D CDA 10-209. The views expressed in this article are those of the authors and do not necessarily reflect the position or policy of the Department of Veterans Affairs, the University of Washington, the University of Michigan or the United States Government. The authors thank the deputy editor and three reviewers for their helpful comments.

Corresponding Author: Edwin S. Wong, Ph.D.; VA Puget Sound Health Care System, Center for Veteran-Centered and Value-Driven Care, 1660 S. Columbian Way, MS S-152, Seattle, WA 98108, USA (e-mail: edwin.wong@va.gov).

\section{Compliance with Ethical Standards:}

Conflict of Interest: Dr. Wong reports ownership of common stock in UnitedHealth Group Inc. and Community Health Systems Inc. All other authors have no conflicts of interest to report.

\section{REFERENCES}

1. Joint principles of the patient-centered medical home. Del Med J. 2008;80(1):21-22.

2. Ferrante JM, Balasubramanian BA, Hudson SV, Crabtree BF. Principles of the patient-centered medical home and preventive services delivery. Ann Fam Med. 2010;8(2):108-116.

3. Kellerman R, Kirk L. Principles of the patient-centered medical home. Am Fam Physician. 2007;76(6):774-775.

4. Kizer KW, Dudley RA. Extreme makeover: Transformation of the veterans health care system. Annu Rev Public Health. 2009;30:313-339.

5. Aysola J, Rhodes KV, Polsky D. Patient-centered medical homes and access to services for new primary care patients. Med Care. 2015;53(10):857-862

6. Shipman SA, Sinsky CA. Expanding primary care capacity by reducing waste and improving the efficiency of care. Health Aff (Millwood). 2013;32(11):1990-1997.

7. Weiner JP, Yeh S, Blumenthal D. The impact of health information technology and e-health on the future demand for physician services. Health Aff (Millwood). 2013;32(11):1998-2004.

8. Helfrich CD, Dolan ED, Simonetti J, et al. Elements of team-based care in a patient-centered medical home are associated with lower burnout among VA primary care employees. J Gen Intern Med. 2014;29(Suppl 2):659-666.

9. Rosland AM, Nelson K, Sun H, et al. The patient-centered medical home in the Veterans Health Administration. Am J Manag Care. 2013;19(7):e263-272.

10. Rich E, Lipson D, Libersky J, Parchman M. Coordinating care for adults with complex care needs in the patient-centered medical home: challenges and solutions: Agency for Healthcare Research and Quality. 2012.

11. Hynes DM, Koelling $\mathbf{K}$, Stroupe $\mathbf{K}$, et al. Veterans' access to and use of Medicare and Veterans Affairs health care. Med Care. 2007;45(3):214-223.

12. Liu CF, Manning WG, Burgess JF Jr, et al. Reliance on Veterans Affairs outpatient care by Medicare-eligible veterans. Med Care. 2011;49(10):911-917.

13. Friedberg MW, Schneider EC, Rosenthal MB, Volpp KG, Werner RM. Association between participation in a multipayer medical home intervention and changes in quality, utilization, and costs of care. JAMA. 2014;311(8):815-825.

14. Hebert PL, Liu CF, Wong ES, et al. Patient-centered medical home initiative produced modest economic results for Veterans Health Administration, 2010-12. Health Aff (Millwood). 2014;33(6):980-987.

15. Reid RJ, Coleman K, Johnson EA, et al. The Group Health medical home at year two: cost savings, higher patient satisfaction, and less burnout for providers. Health Aff (Millwood). 2010;29(5):835-843.

16. Gagne JJ, Glynn RJ, Avorn J, Levin R, Schneeweiss S. A combined comorbidity score predicted mortality in elderly patients better than existing scores. J Clin Epidemiol. 2011;64(7):749-759. 
17. Penfold RB, Zhang F. Use of interrupted time series analysis in evaluating health care quality improvements. Acad Pediatr. 2013;13(6 Suppl):S38-44.

18. Royston P, Ambler G, Sauerbrei W. The use of fractional polynomials to model continuous risk variables in epidemiology. Int $\mathrm{J}$ Epidemiol. 1999;28(5):964-974.

19. Wong ES, Hebert PL, Hernandez SE, et al. Association between local area unemployment rates and use of Veterans Affairs outpatient health services. Med Care. 2014;52(2):137-143.

20. Wong ES, Hebert PL, Nelson KM, et al. Local area unemployment and the demand for inpatient care among Veterans Affairs enrollees. Med Care Res Rev. 2015;72(4):468-480.

21. Dowd BE, Greene WH, Norton EC. Computation of standard errors. Health Serv Res. 2014;49(2):731-750.

22. Elixhauser A, Steiner C, Harris DR, Coffey RM. Comorbidity measures for use with administrative data. Med Care. 1998;36(1):8-27.
23. Southern DA, Quan H, Ghali WA. Comparison of the Elixhauser and Charlson/Deyo methods of comorbidity measurement in administrative data. Med Care. 2004;42(4):355-360.

24. David G, Gunnarsson C, Saynisch PA, Chawla R, Nigam S. Do patientcentered medical homes reduce emergency department visits?. Health Serv Res. 2015;50(2):418-439.

25. Flottemesch TJ, Anderson LH, Solberg LI, Fontaine P, Asche SE. Patient-centered medical home cost reductions limited to complex patients. Am J Manag Care. 2012;18(11):677-686.

26. Higgins S, Chawla R, Colombo C, Snyder R, Nigam S. Medical homes and cost and utilization among high-risk patients. Am J Manag Care. 2014;20(3):e61-71.

27. Mosquera RA, Avritscher EB, Samuels CL, et al. Effect of an enhanced medical home on serious illness and cost of care among high-risk children with chronic illness: a randomized clinical trial. JAMA. 2014;312(24):26402648 